\title{
ORIGINAL ARTICLE Proteomic divergence in Arabidopsis autopolyploids and allopolyploids and their progenitors
}

\author{
DW-K Ng${ }^{1}, C_{\text {Zhang }}{ }^{1}, M_{\text {Miller }}{ }^{1}, Z_{\text {Shen }}^{2}$, SP Briggs ${ }^{2}$ and ZJ Chen ${ }^{1}$
}

Autopolyploidy and allopolyploidy are common in many plants and some animals. Rapid changes in genomic composition and gene expression have been observed in both autopolyploids and allopolyploids, but the effects of polyploidy on proteomic divergence are poorly understood. Here, we report quantitative analysis of protein changes in leaves of Arabidopsis autopolyploids and allotetraploids and their progenitors using isobaric tags for relative and absolute quantitation (iTRAQ) coupled with mass spectrometry. In more than 1000 proteins analyzed, the levels of protein divergence were relatively high ( $\sim 18 \%)$ between Arabidopsis thaliana and Arabidopsis arenosa, relatively low $(\sim 6.8 \%)$ between an $A$. thaliana diploid and autotetraploid and intermediate $(\sim 8.3$ and $8.2 \%)$ in $\mathrm{F}_{1}$ - and $\mathrm{F}_{8}$-resynthesized allotetraploids relative to mid-parent values, respectively. This pattern of proteomic divergence was consistent with the previously reported gene expression data.

In particular, many non-additively accumulated proteins (61-62\%) in the $F_{1}$ and $F_{8}$ allotetraploids were also differentially expressed between the parents. The differentially accumulated proteins in functional categories of abiotic and biotic stresses were overrepresented between an $A$. thaliana autotetraploid and diploid and between two Arabidopsis species, but not significantly different between allotetraploids and their progenitors. Although the trend of changes is similar, the percentage of differentially accumulated proteins that matched previously reported differentially expressed genes was relatively low. Western blot analysis confirmed several selected proteins with isoforms the cumulative levels of which were differentially expressed. These data suggest high protein divergence between species and rapid changes in post-transcriptional regulation and translational modifications of proteins during polyploidization.

Heredity (2012) 108, 419-430; doi:10.1038/hdy.2011.92; published online 19 October 2011

Keywords: polyploidy; proteome; iTRAQ; gene expression; hybrids; Arabidopsis

\section{INTRODUCTION}

Polyploidy has been recognized as a powerful mechanism for evolutionary innovation in many plants and some animals (Wendel, 2000; Doyle et al., 2008; Leitch and Leitch, 2008; Soltis and Soltis, 2009; Wood et al., 2009; Jackson and Chen, 2010). The common occurrence of plant polyploids (including many crops such as wheat, cotton and canola) suggests an evolutionary advantage of having raw genetic materials for selection and adaptation. The molecular basis for this is poorly understood and is probably related to genomic and gene expression changes, which have been demonstrated in many resynthesized and natural polyploid plants, including Arabidopsis (Wang et al., 2004, 2006b), Brassica (Gaeta et al., 2009), Gossypium (Flagel et al., 2008; Hovav et al., 2008), Senecio (Hegarty et al., 2006), Spartina (Salmon et al., 2005) and Tragopogon (Tate et al., 2006; Buggs et al., 2009). These studies have documented several common features of gene expression changes, including non-additive expression, tissuespecific expression of homeologous loci and parental-dependent repression or activation (Jackson and Chen, 2010). In Arabidopsis, the natural allotetraploid Arabidopsis suecica was formed between extant progenitors Arabidopsis thaliana and Arabidopsis arenosa (O'Kane et al., 1995). A previous microarray study revealed a $15 \%$ transcriptome difference between $A$. thaliana and A. arenosa autotetraploids (Wang et al., 2006b). Genome hybridization between these two autotetraploids resulted in 5-6\% transcriptome changes between the resynthesized allotetraploids and their progenitors. In contrast, a very low percentage of transcriptome divergence was observed between an $A$. thaliana diploid and an isogenic autotetraploid line.

Compared with genomic and gene expression changes, changes in proteins and gene products in polyploids and their progenitors are rarely examined. Using protein gel analysis, Birchler and Newton (1981) reported positive and negative effects on protein patterns in maize autoploidy series and aneuploids, with greater effects caused by aneuploidy than by ploidy variation (Birchler and Newton, 1981). Protein two-dimensional electrophoresis (2-DE) analysis in diploid, tetraploid and hexaploid wheat showed that the expression of homeologous proteins in hexaploid wheat depended on interactions among the parental A, B and D genomes (Islam et al., 2003). Other recent studies using 2-DE indicated numerous and non-stochastic modifications of proteins in resynthesized Brassica napus (Albertin et al., 2006, 2009) and wheat hybrids (Song et al., 2007). Using protein 2-DE coupled with mass spectrometry (MS) assays, a recent study showed a positive correlation of differentially expressed proteins with ploidy levels in a maize ploidy series (diploid, tetraploid and hexaploid) (Yao et al., 2011). However, the highest correlations were found in diploid-hexaploid and tetraploid-hexaploid comparisons rather than in the diploid-tetraploid comparison. Notably, differential protein

\footnotetext{
1Section of Molecular Cell and Developmental Biology, Center for Computational Biology and Bioinformatics, and Institute for Cellular and Molecular Biology, The University of Texas at Austin, Austin, TX, USA and 2Division of Biology, University of California, San Diego, La Jolla, CA, USA

Correspondence: Professor ZJ Chen, Institute for Cellular and Molecular Biology, The University of Texas at Austin, One University Station, A-4800, Austin, TX 78712, USA. E-mail: zjchen@mail.utexas.edu
}

Received 9 May 2011; revised 24 August 2011; accepted 31 August 2011; published online 19 October 2011 
regulation did not necessarily correlate with changes in gene expression (Marmagne et al., 2010).

The traditional protein 2-DE has a limited power of detecting the number of protein changes. Isobaric tags for relative and absolute quantitation (iTRAQ) is a suitable method for the study of proteomic changes in polyploids because it is not only capable of simultaneous examination of many more proteins but it also has a high sensitivity for measuring protein abundance in related species when coupled with MS analysis (Ross et al., 2004). iTRAQ-based proteome analysis has been used to identify protein-level differences as small as $50 \%$, and multiple reaction monitoring has been used to confirm these measurements (O'Brien et al., 2010).

Using iTRAQ-based proteomic analysis, in this study, we investigated the effects of genome duplication, divergence and hybridization on proteomic changes in an A. thaliana diploid and an isogenic autotetraploid, between $A$. thaliana and $A$. arenosa autotetraploids, and in A. suecica-like allotetraploids ( $\mathrm{F}_{1}$ and $\mathrm{F}_{8}$ generations) relative to the progenitors. These data provide a general survey of protein regulation in response to changes in genome dosage (diploid vs autotetraploid), genome divergence ( $A$. thaliana vs $A$. arenosa), genome hybridization (allotetraploids relative to the parents) and genetic stability ( $\mathrm{F}_{1}$ vs $\mathrm{F}_{8}$ allotetraploids). In addition, the proteomic data were comparatively analyzed with previous microarray data, and gene ontology (GO) analysis was performed to predict the biological consequences of proteomic changes in these comparisons.

\section{MATERIALS AND METHODS}

\section{Plant materials and growth conditions}

Plant materials included an A. thaliana Landsberg erecta (Ler) diploid (At2), an induced isogenic Ler autotetraploid (At4; ABRC, CS3900), one accession of outcrossing tetraploid A. arenosa (Aa; ABRC, CS3901) (Comai et al., 2000), multiple $\mathrm{F}_{1}$-resynthesized A. suecicalike allotetraploids $\left(\mathrm{AlloF}_{1}\right)$ and one accession (Allo733) of $\mathrm{F}_{8}$ allotetraploids (Allo733 $\mathrm{F}_{8}$ ) (Wang et al., 2006b). The A. thaliana diploid and autotetraploid lines used in this study were the same as those used in previous experiments (Wang et al., 2004, 2006b). A. arenosa is an outcrossing species and is maintained by manual cross-pollination within a small population. Both $A$. arenosa diploids and tetraploids and other hybrid species exist in nature (Koch et al., 2008; Schmickl and Koch, 2011). In the laboratory, allotetraploids could only be resynthesized by pollinating $A$. thaliana autotetraploids with pollen from A. arenosa autotetraploids (Comai et al., 2000). Multiple AlloF 1 lines were generated independently between At 4 and Aa. These lines were self-pollinated to generate stable allotetraploids Allo733 $\left(\mathrm{F}_{6}\right)$, Allo $733 \mathrm{~F}_{7}$ and Allo733F 8 .

Seeds were germinated in Murashige and Skoog agar media with $3 \%$ sucrose (Sigma-Aldrich, St Louis, MO, USA), stratified for 2 days at $4{ }^{\circ} \mathrm{C}$ in the dark and grown at $22^{\circ} \mathrm{C}$ with a $16 / 8$-h (light/dark) cycle in a growth chamber. Two-week-old seedlings were transferred to fresh Murashige and Skoog agar media and grown under the same condition for 6 days before transferring to Pro-mix BX potting soil (Premier Tech Horticulture, Quakertown, PA, USA). For each genotype, mature leaves from a pool of $\geqslant 30$ plants of At 2 and At 4 and 7-8 plants of Aa, $\mathrm{F}_{1}$ and $\mathrm{F}_{8}$ allotetraploids were harvested as one biological replicate. To minimize developmental variation, mature leaves before bolting (from two biological replications) were harvested for protein preparation. This stage was $\sim 4$ weeks (including the time for stratification) in A. thaliana diploids and tetraploids (Ler) under our growth conditions, which is comparable to the growth stage 3.50 in A. thaliana (Col-0), as described previously (Boyes et al., 2001). A similar stage in A. arenosa and the allotetraploids was $\sim 7$ weeks (Wang et al., 2004, 2006b).

\section{Fluorescence in situ hybridization}

To validate the ploidy levels of various plant materials used in this study, fluorescence in situ hybridization was performed according to published protocols (Kato et al., 2004; Lysak et al., 2006a). Flower buds (At2, At4, $\mathrm{Aa}, \mathrm{Allo} 733 \mathrm{~F}_{8}$ ) were fixed in ice-cold Carnoy's fixative for at least $1.5 \mathrm{~h}$. Selected flower buds $(<1 \mathrm{~mm})$ were digested in a pectolytic enzyme mixture $(0.3 \%$ cellulase, $0.3 \%$ pectolyase and $0.3 \%$ cytohelicase) for $2 \mathrm{~h}$. Digested cells were suspended in 9:1 glacial acetic acid:methanol and mounted on glass slides. Chromosomal DNA was cross-linked to slides by exposure to ultraviolet light $\left(120 \mathrm{~mJ} \mathrm{~cm}^{-2}\right)$.

For probe preparation, centromere repetitive sequences of At (AtCEN) and Aa (AaCEN) were amplified by PCR using genomic DNA and the primer pairs as described previously (Comai et al., 2003). PCR products were purified using QIAquick PCR purification kit (Qiagen, Valencia, CA, USA). In all, $1 \mu \mathrm{g}$ of purified AtCEN and AaCEN PCR products were labeled with fluorescein-12-dUTP (Fermentas, Glen Burnie, MD, USA) and Texas red-5-dCTP (Perkin-Elmer, Waltham, MA, USA), respectively, using the nicktranslation method. Labeled probes were purified using MinElute PCR purification kit (Qiagen).

Hybridization of chromosome mounts was performed at $55^{\circ} \mathrm{C}$ for $4-6 \mathrm{~h}$ in the presence of labeled probes. Vectashield mounting medium with DAPI (Vector Laboratories Inc., Burlingame, CA, USA) was applied to hybridized slides before observation. Images were taken using a Zeiss Axiovert $200 \mathrm{~m}$ microscope equipped with a Zeiss Axiocam fluorescence camera (Carl Zeiss Microscopy, LLC, Thornwood, NY, USA).

\section{Flow cytometry}

Endoreduplication is common in many plants, especially in A. thaliana (Galbraith et al., 1983), and the difference in endopolyploidy in leaves of different Arabidopsis lines may contribute to protein expression changes. To determine whether endopolyploidy contributes to proteomic changes among different Arabidopsis lines, flow-cytometric analysis was used to evaluate relative levels of endoreduplication in mature leaves of At2, At4, Aa and Allo733F $\mathrm{F}_{8}$. Mature leaf tissues ( $\sim 150 \mathrm{mg} ; 4-5$ leaves) were collected and placed in a petri dish on ice. Suspension of nuclei was prepared by chopping the plant tissues in a 1-ml buffer ( $\mathrm{pH} 7$ ) containing $45 \mathrm{mM} \mathrm{MgCl}_{2}, 30 \mathrm{~mm}$ sodium citrate, $20 \mathrm{~mm}$ 4-morpholne propane sulfonate and $0.1 \%$ Triton X-100 using a razor blade (Galbraith et al., 1983). Finely chopped leaf tissues were then filtered through a 30- $\mu \mathrm{M}$ Partec CellTrics filter (Partec North America, Swedesboro, NJ, USA) into a $12 \times 75 \mathrm{~mm}^{2}$ polystyrene culture tube. Nucleus suspension was stained by adding $5 \mathrm{mg} \mathrm{ml}^{-1}$ propidium iodide (Sigma-Aldrich) to a final concentration of $100 \mu \mathrm{g} \mathrm{ml}^{-1}$. The nuclei were stained for $40-60 \mathrm{~min}$ before flow cytometry using a BD Biosciences FACSCalibur flow cytometer (BD Biosciences, San Jose, CA, USA). Flow-cytometry data were collected using CellQuestPro software (BD Biosciences). Samples were run under low pressure using the FL2 detector set at $480 \mathrm{~nm}$. A total of $\sim 80000$ events (nuclei) were counted for each sample. Three biological replicates were performed for each line.

Data analysis was performed using the CellQuestPro software, and a scatter plot was generated from the collected data. Populations of nuclei were then identified and gated. Relative proportion of nuclei (from 0,1 and 2 endoreduplication events) within these gated peak regions (FL2-A) were measured and calculated. To determine the degree of endoreduplication in these lines, the average number of endocycles per 100 cells was estimated using the endoreduplication factor (EF) (Cookson et al., 2006). 


\section{Protein extraction and digestion}

For proteomic comparison among the five Arabidopsis lines (At2, At4, $\mathrm{Aa}, \mathrm{AlloF}_{1}$, and $\mathrm{Allo} 733 \mathrm{~F}_{8}$ ), total proteins extracted from mature rosette leaves were used to test the effects of genome duplication (At2 vs At4), genotype difference (At4 vs Aa) and genome hybridization $\left(\mathrm{F}_{1}\right.$ or $\mathrm{F}_{8}$ vs progenitors) on proteome changes. To prepare peptides for iTRAQ reagent labeling, mature leaves $(3 \mathrm{~g})$ of multiple plants from each plant genotype (see above) were ground in liquid nitrogen and washed three times with $50 \mathrm{ml}-20^{\circ} \mathrm{C}$ methanol containing $0.2 \mathrm{mM} \mathrm{Na}_{3} \mathrm{VO}_{4}$ in a $50-\mathrm{ml}$ tube, followed by three washes and precipitation with $50 \mathrm{ml}-20^{\circ} \mathrm{C}$ acetone. Protein pellets were dried in a SpeedVac at $4{ }^{\circ} \mathrm{C}$ and resuspended in $1 \mathrm{ml}$ solution containing $0.2 \mathrm{~mm} \mathrm{Na}_{3} \mathrm{VO}_{4}$ and $0.2 \%$ RapiGest (Waters Corporation, Milford, MA, USA). Supernatants for protein digestion were collected by centrifugation $(16100 \mathrm{~g})$ of samples at $4{ }^{\circ} \mathrm{C}$ for $15 \mathrm{~min}$. Cysteines were reduced and alkylated using $1 \mathrm{~mm}$ Tris (2-carboxyethyl) phosphine (Fisher Scientific, Waltham, MA, USA) at $95^{\circ} \mathrm{C}$ for $5 \mathrm{~min}$ and then $2.5 \mathrm{~mm}$ iodoacetamide (Fisher Scientific) at $37^{\circ} \mathrm{C}$ in the dark for $15 \mathrm{~min}$. Proteins $(100 \mu \mathrm{g})$ were digested with trypsin (Roche, Indianapolis, IN, USA) with a enzyme to substrate ratio (w:w) of 1:50 at $37^{\circ} \mathrm{C}$ overnight.

\section{iTRAQ labeling}

A multiplex iTRAQ shotgun proteomic approach was used to perform quantitative analysis of four samples in a single MS experiment (Ross et al., 2004). The effects of genome duplication on proteomic changes were compared between At 2 and At4. In another multiplexed iTRAQ analysis, proteomes of At4, Aa and two allotetraploids (AlloF 1 and Allo733 $\mathrm{F}_{8}$ ) were compared. The genotypic or species effects on proteomes were analyzed between an induced $A$. thaliana autotetraploid (At4) and natural A. arenosa tetraploids (Aa). For simplicity, we use the terms 'genotype' and 'species' interchangeably between these comparisons. Finally, genome hybridization effects were compared between either AlloF $F_{1}$ or Allo733F $\mathrm{F}_{8}$ and their progenitors (At4 and Aa). In a single MS experiment, proteomes from four samples were analyzed simultaneously using four iTRAQ reagents (AB SCIEX, Foster City, CA, USA) containing different reporter groups of mass to charge ratio $(\mathrm{m} / \mathrm{z})$ of $114,115,116$ or 117 . In comparing diploid and autotetraploid A. thaliana, iTRAQ 114 and 115 reagents were used to label tryptic-digested peptides from At2, and iTRAQ 116 and 117 reagents were used for At4 tryptic-digested peptides labeling. In another multiplexed iTRAQ analysis, tryptic-digested peptides from At4, Aa, AlloF $F_{1}$ and Allo733 $\mathrm{F}_{8}$ allotetraploids were labeled with iTRAQ reagents with $m / z$ of $114,115,116$ and 117, respectively. Each peptide solution was reacted with one-half tube of iTRAQ reagent in 90\% isopropyl alcohol and $250 \mathrm{~mm}$ HEPES (4-(2-hydroxyethyl)-1piperazineethanesulfonic acid) at a $\mathrm{pH}$ of 7.2 for $15 \mathrm{~min}$ at room temperature. Water was then added to a final isopropyl alcohol concentration of $60 \%$. The second half tube of the iTRAQ reagent was added and samples were incubated at room temperature for $2 \mathrm{~h}$. Peptides were dried in a vacuum concentrator and resuspended in water $(100 \mu \mathrm{l})$. Samples tagged with different iTRAQ reagents were pooled together. Thereafter, $1 \%$ trifluoroacetic acid (final $\mathrm{pH}<2$ ) was added to precipitate RapiGest. Samples were incubated at $4{ }^{\circ} \mathrm{C}$ overnight and then centrifuged at $16100 \mathrm{~g}$ for $15 \mathrm{~min}$. The supernatants were collected and centrifuged through a $0.22-\mu \mathrm{M}$ filter for subsequent liquid chromatography (LC)-MS/MS analysis.

\section{Three-phase online nano-LC}

The iTRAQ-labeled peptides were separated into fractions using nano-LC for subsequent MS analysis. Nano-LC was performed with an Agilent 1100 HPLC system (Agilent Technologies, Santa Clara, CA, USA) using the exact conditions and columns as described previously (O'Brien et al., 2010). A total of 28 salt fractions were collected for each full proteome analysis.

\section{Tandem MS}

The collected peptide fractions from nano-LC were subjected to tandem MS for peptide identification and quantitation. A LTQ linear ion trap tandem mass spectrometer (Thermo Electron Corporation, San Jose, CA, USA) was used to acquire spectra under automated, data-dependent acquisition. MS was performed according to the conditions as described previously (O'Brien et al., 2010). iTRAQ reagents were originally not usable in ion trap instruments because of the 'one-third rule'. To illustrate, fragment ions of a $\mathrm{m} / \mathrm{z} 900$ parent will not be detected below $m / z 300$, and this would normally prevent the detection of iTRAQ reporter ions. To overcome this limit, a new collision-activated fragmentation technique, called pulsed-Q dissociation (PQD), was used to enable routine and reliable measurement of ions down to $50 \mathrm{~m} / \mathrm{z}$ (Schwartz et al., 2005; Griffin et al., 2007). For peptide identification and quantitation, collision-induced dissociation and PQD scans were collected for the same parent ion (Andreyev et al., 2010; O'Brien et al., 2010). For each MS scan, four pairs of collision-induced dissociation-PQD MS/MS scans were performed for the most intense ions from the parent MS scan. Dynamic exclusion was set at $1 \mathrm{~min}$ to improve the duty cycle of MS/MS scans. Approximately $20000 \mathrm{MS} / \mathrm{MS}$ spectra were generated and collected for each salt step fractionation. We have developed and successfully applied a combined collision-induced dissociation/PQD scan approach for peptide identification and quantitation (Andreyev et al., 2010; O’Brien et al., 2010).

\section{Protein database search}

To infer protein identities from the identified peptides from MS/MS spectra, the raw data were extracted and searched using Spectrum Mill v3.03 (Agilent Technologies). Both collision-induced dissociation and PQD scans from the same parent ion were merged together. MS/MS spectra with a sequence tag length $>1$ were searched against a concatenated forward-decoy database (71238 protein sequences). The concatenated forward-decoy database was constructed by combining the International Protein Index Arabidopsis database (v3.29, 35619 protein sequences) and a 1:1 decoy database. The decoy database was constructed by first reversing the International Protein Index protein sequences. The tryptic peptides (peptide length $>6$ ) shared with the forward International Protein Index database were then shuffled to ensure that there were no shared tryptic peptides between the forward and decoy databases. Fully tryptic peptides with a maximum miscleavage of one setting were used as the enzyme parameter for the database search. All other search parameters were set to default settings of Spectrum Mill (carbamidomethylation of cysteines, iTRAQ modification, $\pm 2.5 \mathrm{kDa}$ for precursor ions, $\pm 0.7 \mathrm{kDa}$ for fragment ions and a minimum matched percentage score peak intensity of $50 \%$ ). The in situ false discovery rate was derived with the constructed concatenated forward-decoy database. To maintain the false discovery rate of protein levels at $<1 \%$, a MS/MS spectrum $>50 \%$ score peak intensity and cutoff scores $>15.7,>13.7$ and $>15.0$ were dynamically assigned to the data set with $1+, 2+$ and $3+$ peptides, respectively. Proteins with two or more unique peptides were validated and used for quantitative analysis. To address the issue of database redundancy resulting from proteins with high sequence homology or alternative splice variants, common peptides shared between proteins were grouped such that proteins within the same 
group shared the same set or subset of unique peptides. However, if a protein with shared peptides also contained other unique peptides, it was assigned to a different protein group. Within a protein group, the protein with the highest total identification score of all peptides was chosen as the group leader for proteomic comparisons among all Arabidopsis lines used in this study.

\section{Protein quantification by iTRAQ mass tagging reagent}

For quantitative analysis of proteins, shared peptides between protein groups were removed, and unique peptides from a protein group were used for iTRAQ quantitation. Relative protein quantitation between different Arabidopsis lines was performed by calculating the iTRAQ reporter ion intensity ratios of respective lines. Protein iTRAQ intensities were derived by summing the peptide iTRAQ intensities from each protein group. Low-intensity spectra with total iTRAQ reporter intensity (sum of all 4 channels) $<100$ were filtered. Isotope impurities of iTRAQ reagents were corrected according to the manufacturer's instructions (AB SCIEX). For protein iTRAQ reporter intensities normalization, log ratios between different iTRAQ tags $(115 / 114,116 / 114,117 / 114)$ were adjusted globally to attain a median $\log$ ratio of zero. The normalized protein iTRAQ intensities were used for quantitative analysis.

\section{Quantitative proteome comparison between different Arabidopsis lines}

Quantitative protein differences were estimated between autotetraploid and diploid A. thaliana (At4/At2), A. arenosa and A. thaliana (Aa/At4) autotetraploids, the allotetraploids AlloF 1 or Allo733F $\mathrm{F}_{8}$ and the mid-parent value (MPV; $1 / 2$ At $4+1 / 2$ Aa) $\left(\mathrm{F}_{1} / \mathrm{MPV}\right.$ or $\left.\mathrm{F}_{8} / \mathrm{MPV}\right)$. For each comparison, the normalized protein iTRAQ intensities from two biological replicates were used. Only proteins detected in both MS experiments were used to calculate the protein fold change. Student's $t$-tests were performed to determine protein expression differences between each comparison in two replications $(P \leqslant 0.05)$. $P$-value corrections for multiple testing were performed using the SGoF software (Carvajal-Rodriguez et al., 2009). In addition to Student's $t$-test $P$-values, we adjusted $P$-values from various multiple testing methods, including SGoF (Carvajal-Rodriguez et al., 2009), Benjamini and Hochberg (Benjamini and Hochberg, 1995), SGoF plus (de UnaAlvarez and Carvajal-Rodriguez, 2010), SFisher (Diz et al., 2011) and Bonferroni (Holm, 1979). In addition, a complete list of protein quantification data is provided in Supplementary Data Set 1 (http:// datadryad.org), in which independent $P$-value adjustments could be performed. The multiple testing results are provided in Supplementary Tables S2-S5. In Supplementary Table S6, we also provide a comparison of the numbers of differentially expressed proteins under each of the correction methods used. All proteins were sorted according to their significance levels. For each comparison (At4/At2, Aa/At4, $\mathrm{F}_{1} / \mathrm{MPV}$ or $\mathrm{F}_{8} / \mathrm{MPV}$ ), preference in upregulation or downregulation of proteins was evaluated using Wilcoxon's signed-rank tests.

Although $P$-value corrections for multiple comparisons would decrease the probability of false positives (type I errors), the probability of false negatives (type II errors) would increase depending on which multiple hypothesis testing methods were used (Diz et al., 2011). In this study, many proteins including two that were validated by western blots were excluded in several corrections for multiple comparisons. In some tests, the number of significant proteins was zero. As the major goal of this study is to survey the overall proteomic changes among different Arabidopsis lines, proteins in comparison with the uncorrected $P$-value $(\leqslant 0.05)$ were used for further analysis. Although this would potentially increase false positives, such occurrence could be corrected by future experimental validation for the proteins of interest. Moreover, the proteomic data in this study were comparatively analyzed using the previously reported transcriptome (microarray) data (Wang et al., 2006b).

\section{GO analysis}

To determine whether the differentially accumulated proteins among different Arabidopsis lines were associated with particular biological functions, GO analysis was performed using the online database at The Arabidopsis Information Resource website (Berardini et al., 2004), and proteins were grouped into 14 biological process categories. GO enrichment of all proteins detected from the two iTRAQ experiments were compared against the whole Arabidopsis genome categorization in The Arabidopsis Information Resource website. $\chi^{2}$ Tests were used to determine the significance of GO enrichment for proteins detected in the iTRAQ experiments. GO enrichments of differentially expressed proteins resulting from genome duplication (At4 vs At2), genotype difference (Aa vs At4) and genome hybridization $\left(\mathrm{AlloF}_{1}\right.$ or Allo733F $\mathrm{F}_{8}$ vs MPV) were also compared and the significance of enrichment was determined using $\chi^{2}$ tests.

\section{Extraction of nuclei and thylakoid proteins}

To validate histone protein accumulation in various Arabidopsis lines, crude nuclei were prepared from the same plant materials for protein extraction and western blots. Rosette leaves from multiple plants of a single line $(400 \mathrm{mg})$ were ground in the presence of liquid nitrogen, and the frozen powder was transferred to a test tube containing $1 \mathrm{ml}$ of nuclei isolation buffer with $0.25 \mathrm{M}$ sucrose, $15 \mathrm{~mm}$ Piperazine- $N, N^{\prime}$-bis (2-ethane-sulfonic acid) ( $\mathrm{pH} 6.8$ ), $5 \mathrm{~mm} \mathrm{MgCl}_{2}, 60 \mathrm{mM} \mathrm{KCl}, 15 \mathrm{~mm}$ $\mathrm{NaCl}, 1 \mathrm{~mm} \mathrm{CaCl}_{2}, 0.9 \%$ Triton X-100, $2 \mu \mathrm{g} \mathrm{ml}^{-1}$ Pepstatin A, $1 \mathrm{~mm}$ PMSF (phenylmethanesulfonyl fluoride) and $1 \times$ protease inhibitor cocktail (Sigma-Aldrich) (Saleh et al., 2008). The samples were incubated on ice for $15-30 \mathrm{~min}$, filtered through one layer of Miracloth and subjected to centrifugation at $11000 \mathrm{~g}$ for $20 \mathrm{~min}$ at $4{ }^{\circ} \mathrm{C}$. The pellet was resuspended in $100 \mu \mathrm{l}$ of cold nucleus lysis buffer containing $50 \mathrm{~mm}$ HEPES ( $\mathrm{pH} 7.5$ ), $150 \mathrm{~mm} \mathrm{NaCl}, 1 \mathrm{~mm}$ EDTA, $1 \mathrm{~mm}$ PMSF, $1 \%$ SDS, $1 \%$ Triton X-100, $1 \mu \mathrm{g} \mathrm{ml}^{-1}$ pepstatin A and $1 \times$ protease inhibitor cocktail (Sigma-Aldrich).

To validate an Arabidopsis leaf-type ferredoxin-NADP oxidoreductase (AtLFNR1; At5g66190) accumulation in different Arabidopsis lines, thylakoid proteins from rosette leaves $(400 \mathrm{mg})$ were prepared according to a published protocol (Lintala et al., 2007). In brief, frozen tissues were ground and incubated for $30 \mathrm{~min}$ in $500 \mu \mathrm{l}$ of grinding buffer containing $10 \mathrm{~mm}$ HEPES ( $\mathrm{pH} 7.6$ ), $5 \mathrm{~mm}$ sucrose, $5 \mathrm{~mm} \mathrm{MgCl}$, $2 \mathrm{~mm}$ PMSF and $1 \times$ protease inhibitor cocktail (Sigma-Aldrich). The samples were filtered through one layer of Miracloth and subjected to centrifugation $(2500 \mathrm{~g})$ at $4{ }^{\circ} \mathrm{C}$ for $4 \mathrm{~min}$. The pellet (thylakoids) was resuspended in $100 \mu \mathrm{l}$ of storage buffer containing $10 \mathrm{mM}$ HEPES ( $\mathrm{pH}$ 7.5), 100 mм sucrose, $5 \mathrm{~mm} \mathrm{NaCl}, 10 \mathrm{~mm} \mathrm{MgCl}_{2}, 2 \mathrm{~mm}$ PMSF and $1 \times$ protease inhibitor cocktail (Sigma-Aldrich).

\section{SDS-PAGE and western blot analysis}

We validated two proteins of the proteomic data using western blot analysis because protein antibodies are very limited. For western analysis, an aliquot $(20 \mu \mathrm{g})$ of nuclei or thylakoid protein was subjected to $15 \%$ SDS-PAGE (6m urea) and transferred onto a polyvinylidene fluoride membrane (GE Healthcare, Piscataway, NJ, USA). An aliquot $(1 \mu \mathrm{g})$ of core histones (Millipore, Billerica, MA, USA) was included as a positive control in the western blot analysis of histone H3. For western blotting, the membrane was incubated at room temperature for $1.5 \mathrm{~h}$ in a protein-free blocking 
buffer (Thermo Scientific, Waltham, MA, USA) containing 0.05\% Tween-20. The primary antibodies, anti-H3Cter (1:1000) (Abcam, Cambridge, MA, USA) and anti-AtLFNR1 (1:3000) (Abcam), were used to probe the immunoblots containing nuclei and thylakoid protein extracts, respectively. The goat anti-rabbit IgG, horseradish peroxide conjugate (1:10000) (Millipore) was used as a secondary antibody for immunodetection, which was performed using a SuperSignal West Pico kit (Thermo Scientific) and quantified using ImageJ software (National Institutes of Health, Bethesda, MD, USA).

\section{RESULTS}

Chromosomal and endopolyploidy analyses in A. thaliana diploid (At2), tetraploid (At4), A. arenosa autotetraploid (Aa) and allotetraploid (Allo733F 8 )

The iTRAQ-based proteomic analysis was performed using rosette leaves (from multiple plants) of various Arabidopsis lines: (1) A. thaliana (Ler ecotype) diploid (At2); (2) an isogenic A. thaliana autotetraploid (At4); (3) natural A. arenosa autotetraploids (Aa); (4) newly resynthesized $A$. suecica-like $\mathrm{F}_{1}\left(\mathrm{AlloF}_{1}\right)$ lines and (5) a stable A. suecica-like allotetraploid (Allo733 $\mathrm{F}_{8}$ ) (Figures 1a-d). Fluorescence in situ hybridization confirmed the ploidy levels of these lines with 10 , 20 and 32 chromosomes in At2, At4 and Aa, respectively (Figures 1eg). Allo733 $\mathrm{F}_{8}$ contained 26 chromosomes, including 10 and 16 from A. thaliana and A. arenosa, respectively (Figure 1h) (Comai et al., 2000; Wang et al., 2006b).

To address possible complicating effects of endopolyploidy (Galbraith et al., 1983) on interpreting patterns of proteomic divergence, flow-cytometric analysis was used to evaluate relative levels of endoreduplication in At2, At4, Aa and Allo733F $\mathrm{F}_{8}$ (Figures 2a-d). The distribution of $2 \mathrm{C}, 4 \mathrm{C}$ and $8 \mathrm{C}$ nuclei in the diploid (At2) was shifted proportionally to the corresponding $4 \mathrm{C}, 8 \mathrm{C}$ and $16 \mathrm{C}$ nuclei in tetraploids (At4) (Figure 2e; Supplementary Table S1). At4 showed an overall higher EF (more cycles of endoreduplication) than did At2 in leaves (Figure 2f; $P=0.01$ ), indicating that endopolyploidy levels increase proportionally in response to ploidy changes. Among the various Arabidopsis tetraploids, At4, Aa and Allo733 $\mathrm{F}_{8}$ had different endopolyploidy distribution patterns (Figure 2e). Overall, Aa showed a lower EF than did At4 $(P=0.001)$ or Allo733F $(\mathrm{F} 8)(P=0.011)$ (Figure 2f), and no significant difference was observed between At4 and Allo733F $\mathrm{F}_{8}$ allotetraploids $(P=0.528)$. These data indicate that endopolyploidy levels in leaves were low in A. arenosa but similar between an $A$. thaliana autotetraploid and $\mathrm{F}_{8}$ allotetraploid and increased proportionally between an $A$. thaliana diploid and autotetraploid.

\section{Proteomic divergence in Arabidopsis-related species and allotetraploids}

In two repeated iTRAQ LC-MS/MS analyses, a total of 80753 MS/MS spectra were validated. Among them, 131 spectra were from the decoy database, resulting in a false discovery rate of $0.16 \%$ at the spectrum level. In addition, 8945 unique peptides were identified with a false discovery rate of $0.65 \%$ at the peptide level (58 peptides from the decoy database). A total of 2083 common proteins were identified by two replications of iTRAQ and MS assays between At2 and At4. After analyzing shared peptides, these proteins were classified into 1400 groups. Among them, 95 proteins $(\sim 6.8 \%)$ displayed expression differences $(P \leqslant 0.05)$ between At 2 and At4 (Figures $3 \mathrm{a}$ and $c$ ). Between various Arabidopsis tetraploids (At4, Aa, AlloF ${ }_{1}$ and Allo733 $\mathrm{F}_{8}$ ), a total of 1160 protein groups were identified from 1730 common proteins that were detected in the 2 replicated iTRAQ and MS assays. The proteomic divergence between At4 and Aa was relatively high, and 209 of the 1160 proteins $(\sim 18 \%)$ present in both replicates showed expression divergence between $A$. thaliana and A. arenosa (Figures $3 \mathrm{~b}$ and $\mathrm{c}$ ). In the allotetraploids that were derived
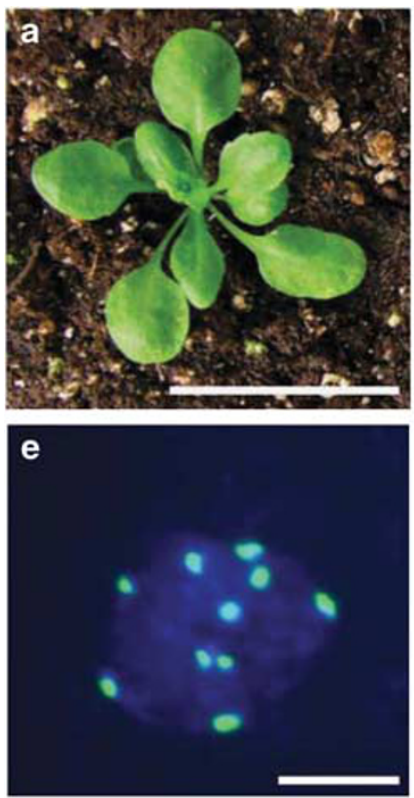

A. thaliana $(2 \mathrm{n}=2 \mathrm{x}=10)$
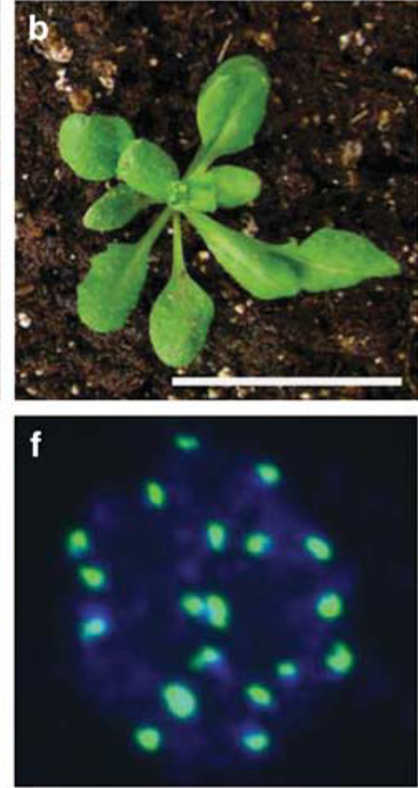

A. thaliana $(2 n=4 x=20)$
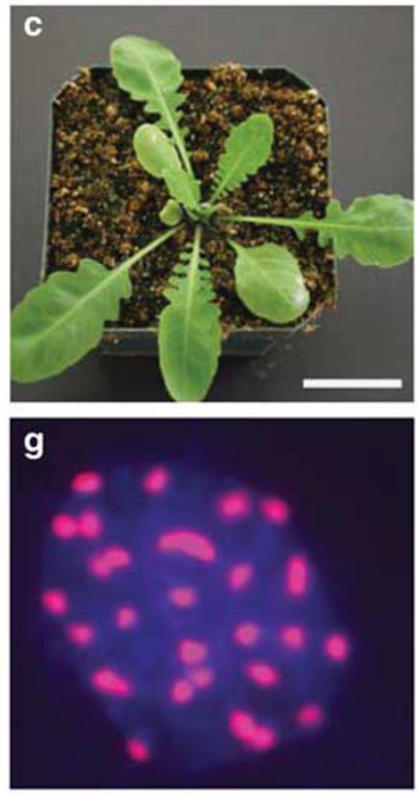

A. arenosa $(2 \mathrm{n}=4 \mathrm{x}=32)$
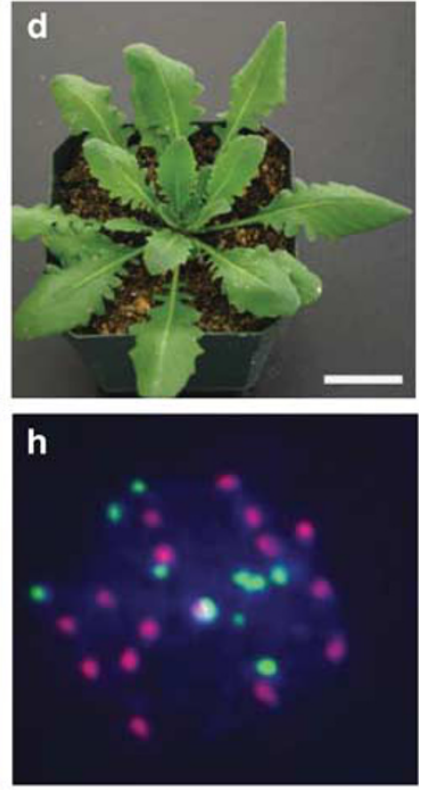

Allo733 $(2 n=4 x=26)$

Figure 1 Plant materials and fluorescence in situ hybridization (FISH). (a, e) Arabidopsis thaliana (Ler) diploid (At2; $2 n=2 x=10)$. (b, f) $A$. thaliana autotetraploid (At4; $2 n=4 x=20$ ). (c, g) Arabidopsis arenosa autotetraploid (Aa; $2 n=4 x=32$ ). (d, h) Resynthesized allotetraploid (Allo733F 8 ; $2 n=4 x=26$ ) derived from $A$. thaliana and $A$. arenosa. Fluorescein-labeled (green) and Texas red-labeled (Red) probes were used to detect At and Aa centromeric repeats, respectively. Bars $=5 \mu \mathrm{m}$ (panels a-d) and $4 \mathrm{~cm}$ (panels e-h). 

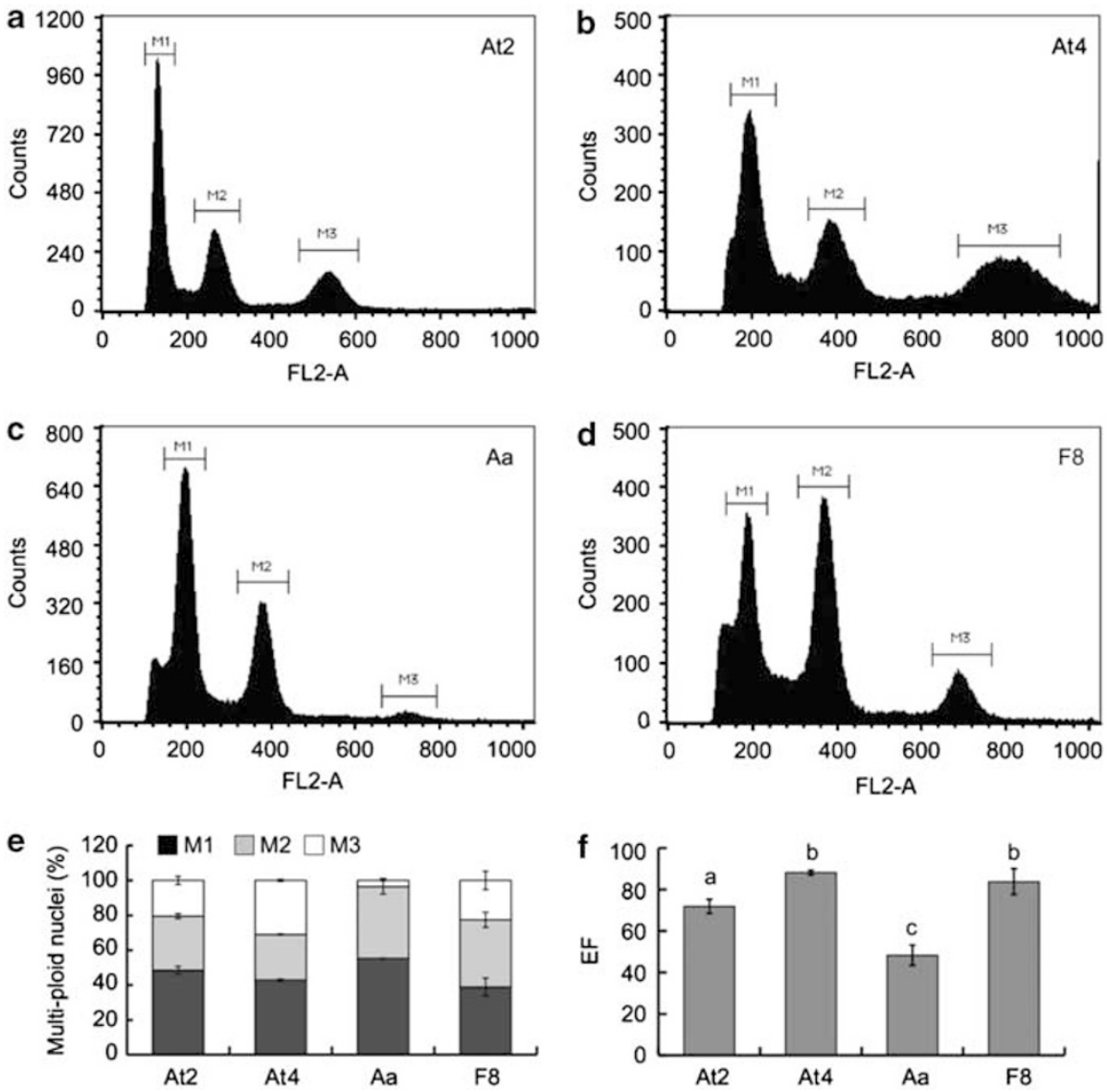

Figure 2 Flow-cytometric analysis of endopolyploidy in mature leaves of different Arabidopsis lines. (a-d) Flow-cytometry histograms of gated nuclei fluorescence detected using the FL2 $(480 \mathrm{~nm}$ ) photodetector (FL2-A) using mature leaves of an $A$. thaliana diploid (panel a), A. thaliana autotetraploid (panel b), A. arenosa tetraploid (panel c) and allotetraploid Allo733 in the $\mathrm{F}_{8}$ generation $\left(\mathrm{Allo} 733 \mathrm{~F}_{8}\right.$ ) (panel d). Markers (M1 to M3) indicate 0,1 and 2 endoreduplication events, respectively. (e) The proportion of cell ploidy levels $(n=3)$. M1, M2 and M3 represent $2 \mathrm{C}, 4 \mathrm{C}, 8 \mathrm{C}$ nuclei in a diploid, respectively, and $4 \mathrm{C}, 8 \mathrm{C}$ and $16 \mathrm{C}$ nuclei in a tetraploid, respectively. (f) The endoreduplication factor (EF) represents the average endocycles per 100 cells $(n=3)$ in the 4 lines examined. Error bars indicate s.e. The significance levels $(P \leqslant 0.05)$ are shown as ' $a, b$ and $c$ ' in multiple comparison tests.

from A. thaliana and A. arenosa, $97(\sim 8.4 \%)$ and $95(\sim 8.2 \%)$ proteins in $\mathrm{F}_{1}$ and $\mathrm{F}_{8}$ allotetraploids, respectively, showed non-additive abundance relative to the MPV of At4 and Aa, respectively.

Many non-additively expressed proteins in allotetraploids were also differentially expressed between the parents. Among the non-additively accumulated proteins in $\mathrm{F}_{1}$ and $\mathrm{F}_{8}$ allotetraploids, approximately 61-62\% were also differentially expressed between the progenitors (Figure 3d). A total of 151 proteins were non-additively expressed in $\mathrm{F}_{1}$ and $\mathrm{F}_{8}$ allotetraploids, and $41(\sim 27 \%)$ of them were shared between the 2 allotetraploids (Figure $3 \mathrm{~d}$ ).

\section{Effects of genome dosage and hybridization on proteome divergence in Arabidopsis}

Among the differentially or non-additively expressed proteins, 14 of 95 differentially expressed proteins $(\sim 14.7 \%)$ between diploid and autotetraploid A. thaliana showed \pm 2 -fold difference (Figure $4 \mathrm{a}$ ). Among them, eight proteins were upregulated twofold or more, and six were downregulated twofold or more in the autotetraploid. Overall, the numbers of upregulation or downregulation of proteins were similar between an A. thaliana diploid and an isogenic autotetraploid.

Between A. thaliana and A. arenosa autotetraploids, 158 ( $\sim 75.6 \%)$ of 209 differentially expressed proteins displayed \pm 2 -fold expression difference (Figure $4 \mathrm{~b}$ ). Among them, 67 ( $\sim 42.4 \%)$ proteins accumulated at higher levels in A. arenosa than in A. thaliana, whereas 91
( $\sim 57.6 \%)$ accumulated at higher levels in $A$. thaliana than in A. arenosa (Supplementary Table S3). A slightly larger proportion of non-additively expressed proteins, $\sim 56.7 \%$ (55 of 97 ) and $\sim 53.7 \%$ (51 of 95), respectively, were downregulated in $\mathrm{F}_{1}$ and $\mathrm{F}_{8}$ allotetraploids (Figures 4c and d). Moreover, 24.7\% (24 of 97) and $~ 28.4 \%$ (27 of 95) of the non-additively accumulated proteins, respectively, were downregulated by $\geqslant 2$-fold. However, the differences in upregulation or downregulation of proteins within each comparison (Aa/At4, $\mathrm{F}_{1} / \mathrm{MPV}$ or $\mathrm{F}_{8} / \mathrm{MPV}$ ) were not statistically significant.

Finally, among the non-additively accumulated proteins in $\mathrm{F}_{1}$ and $\mathrm{F}_{8}$ allotetraploids, expression changes for the majority of proteins were in the same direction; 16 were upregulated, 22 downregulated and 3 in opposing directions (Supplementary Tables S4 and S5). The data suggested that proteomic changes in selfed progeny of polyploids are relatively stable.

Enrichment of protein functional groups in response to genome duplication, divergence and hybridization

Total proteins identified by the iTRAQ LC-MS/MS analysis were grouped into $14 \mathrm{GO}$ biological process categories based on The Arabidopsis Information Resource GO annotation (Berardini et al., 2004). Compared with the proportion of A. thaliana whole-genome proteins, proteins related to stress, abiotic or biotic stimulus, electron transport and energy pathways were significantly enriched (>300\%) 


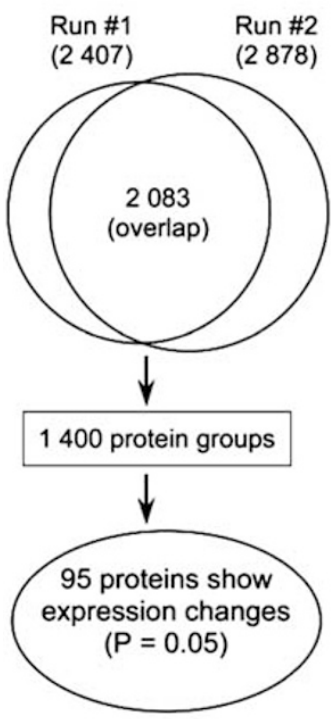

b Run \#1

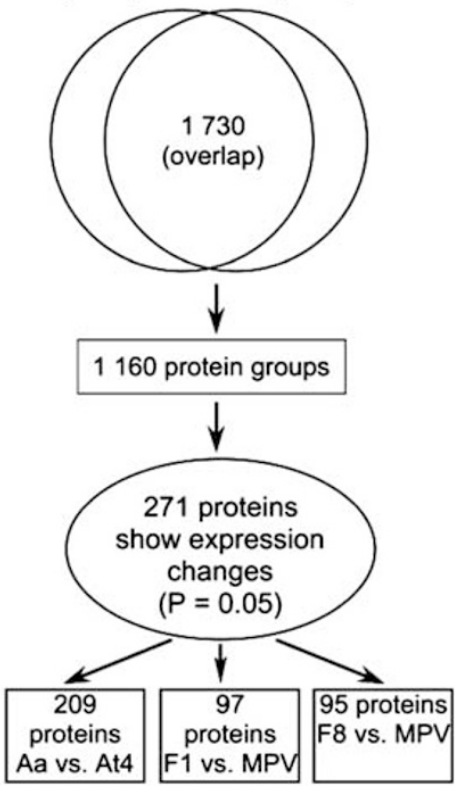

C

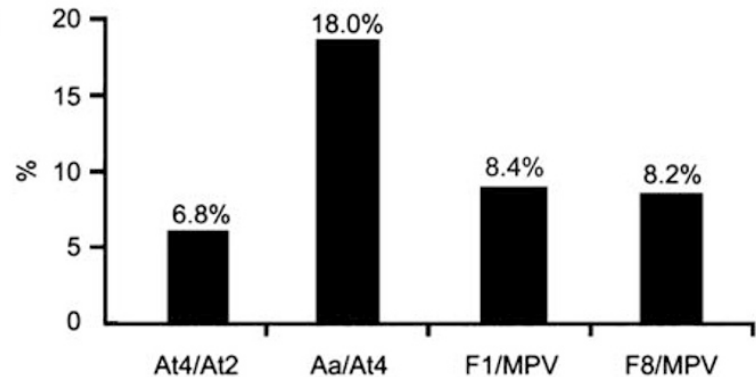

d

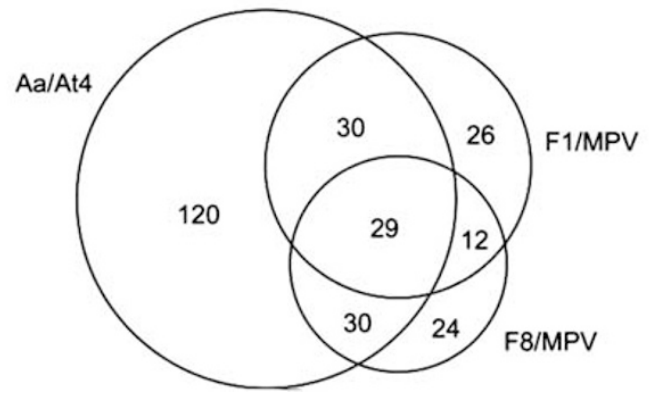

Figure 3 Use of iTRAQ strategy for analysis of proteome divergence and non-additive accumulation in Arabidopsis-related species. (a) Proteome comparison between $A$. thaliana (Ler) diploids (At2) and autotetraploids (At4). (b) Proteome comparison between $A$. thaliana autotetraploids (At4), $A$. arenosa autotetraploids $(\mathrm{Aa})$ and their allotetraploid hybrids in the $\mathrm{F}_{1}$ and $\mathrm{F}_{8}$ generations. Two LC-MS/MS runs were performed for each comparison. Proteins identified in both runs (overlap) were used for expression divergence analysis. Proteins sharing the same peptides were assigned to a protein group, and the protein with the highest identification score designated as the group leader for proteomic comparisons among all Arabidopsis species. Protein expression differences were determined using a significance level of $P \leqslant 0.05$ within each comparison. MPV, mid-parent value $(1 / 2 A t 4+1 / 2 A a)$. (c) Percentage of proteins showing expression divergence between $A$. thaliana (Ler) diploids (At2) and tetraploids (At4), $A$. thaliana and $A$. arenosa tetraploids (Aa/At4), and resynthesized $F_{1}$ and

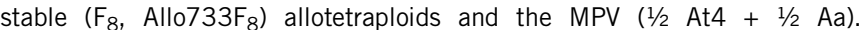
(d) Venn diagram showing the number of differentially accumulated proteins between $\mathrm{Aa}$ and At4 and non-additively accumulated proteins between $\mathrm{F}_{1}$ or $\mathrm{F}_{8}$ allotetraploids and the MPV.
(Figure 5a). Proteins that belong to cell organization and biogenesis, protein metabolism and other biological processes were also overrepresented (150-280\%). In contrast, proteins of the transcription and signal transduction categories were significantly underrepresented. No significant GO enrichments were found for proteins within the categories of developmental processes and DNA/RNA metabolism.

In comparison with differentially expressed proteins between A. thaliana diploids (At2) and autotetraploids (At4), GO categories of stress responses $(\sim 175 \%)$ and abiotic or biotic stimulus $(\sim 178 \%)$ were significantly enriched ( $\chi^{2}$ test, $P \leqslant 0.05$ ) (Figure $5 b$ ). Although proteins within electron transport and energy pathways were enriched ( $\sim 197 \%), \chi^{2}$ tests revealed no significant difference. Within the stress response category, 25 of 37 proteins accumulated at higher levels in At4 than in At2. A similar trend was observed for proteins in response to the abiotic or biotic stimulus category; 24 of 36 proteins accumulated at higher levels in At4 than in At2 (Supplementary Figure S1; Supplementary Table S2). Although the proteomes between A. thaliana and A. arenosa showed the highest divergence, enrichment of differentially expressed proteins was modest among GO categories. Significant enrichment was found only for proteins related to the response to abiotic or biotic stimulus (Figure 5b). In resynthesized allotetraploids, non-additively expressed proteins involved in developmental processes, abiotic/biotic stimulus response and electron transport/energy pathways were relatively enriched (approximately 123-141\%). Among differentially accumulated proteins, proteins in the transcription (in $\mathrm{F}_{1}$ ) and DNA/RNA metabolism (in $\mathrm{F}_{1}$ or $\mathrm{F}_{8}$ ) categories were overrepresented by $\sim 190 \%$ or more. However, these categories only accounted for a low proportion of proteins that were identified by iTRAQ (Figure $5 \mathrm{a}$ ). No significant enrichment was found for any of the GO categories in either $\mathrm{F}_{1} / \mathrm{MPV}$ or $\mathrm{F}_{8} / \mathrm{MPV}$ comparison.

Comparison of proteomic with transcriptomic data

In comparison with a previously reported microarray analysis (Wang et al., 2006b), 8 ( 8.4\%), $125(\sim 59.8 \%)$ and 44 ( $\sim 46.3 \%)$ differentially or non-additively expressed proteins in this study also showed transcript level differences in At4/At2, Aa/At4 and $\mathrm{F}_{8} / \mathrm{MPV}$, respectively (Figure 6a). Similar upregulation or downregulation of transcript and protein levels suggests transcriptional regulation of gene expression. Between At4 and At2, five genes were upregulated at both transcript and protein levels (Supplementary Table S2). These included pathogenesis-related protein 5 (At1g75040), $\beta$-carbonic anhydrase 2 (At5g14740), TSK-associating protein 1 (At1g52410), mitochondrial alanyl-tRNA synthetase (Atlg50200) and ribosomerecycling factor (At3g63190). The transcriptomic and proteomic comparison between At 4 and Aa revealed 32 (25.6\%) and 34 $(27.2 \%)$ proteins displaying the same trend of upregulation and downregulation, respectively (Supplementary Table S3). In $\mathrm{F}_{8}$ allotetraploids, $8(\sim 18.2 \%)$ and $18(\sim 40.9 \%)$ proteins shared the same trend of upregulation and downregulation, respectively (Supplementary Table S5). In the proteomic analysis, a major latex protein-like protein, major latex protein-like 423 (Atlg24020), was highly expressed in $A$. arenosa (Aa/At4 $=4.322 \pm 0.318$ ) but downregulated in the allotetraploids $\left(\mathrm{F}_{1} / \mathrm{MPV}=0.437 \pm 0.074 ; \quad \mathrm{F}_{8} /\right.$ $\mathrm{MPV}=0.306 \pm 0.066$ ), which was consistent with the microarray data of upregulation or downregulation at the transcript levels (Supplementary Tables S3 and S5). Interestingly, similar findings were found in resynthesized B. napus allotetraploids (stem tissues) using protein two-dimensional analysis (Albertin et al., 2006) and semi-quantitative reverse transcriptase-PCR assays (Marmagne et al., 2010). Finally, expression of $3(\sim 37.5 \%), 59(\sim 47.2 \%)$ and 18 

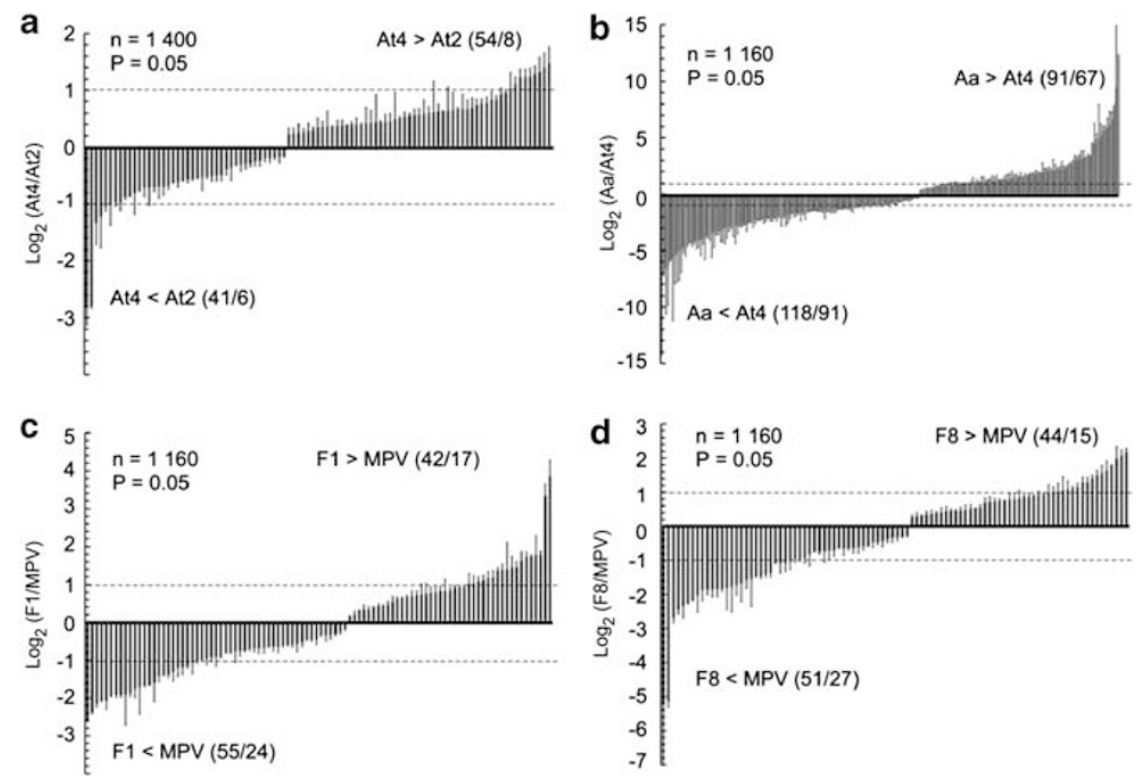

Figure 4 Protein expression changes in Arabidopsis autotetraploids and allotetraploids and their progenitors. Histograms showing expression levels (in log 2 scale) of differentially expressed proteins between (a) $A$. thaliana diploids and autotetraploids; (b) $A$. arenosa and $A$. thaliana tetraploids; (c) $\mathrm{F}_{1}$-resynthesized allotetraploids and the MPV; and $(\mathbf{d}) \mathrm{F}_{8}$-resynthesized allotetraploids $\left(A \mathrm{Alo} 733 \mathrm{~F}_{8}\right.$ ) and the MPV. The number of downregulated or upregulated proteins is showed in parenthesis. Error bars showed the s.d. of the log ratio. Dashed lines mark the \pm 2 -fold change levels, respectively. MPV: mid-parent value. Total numbers of differentially expressed proteins over those with \pm 2 -fold changes are shown in parentheses.

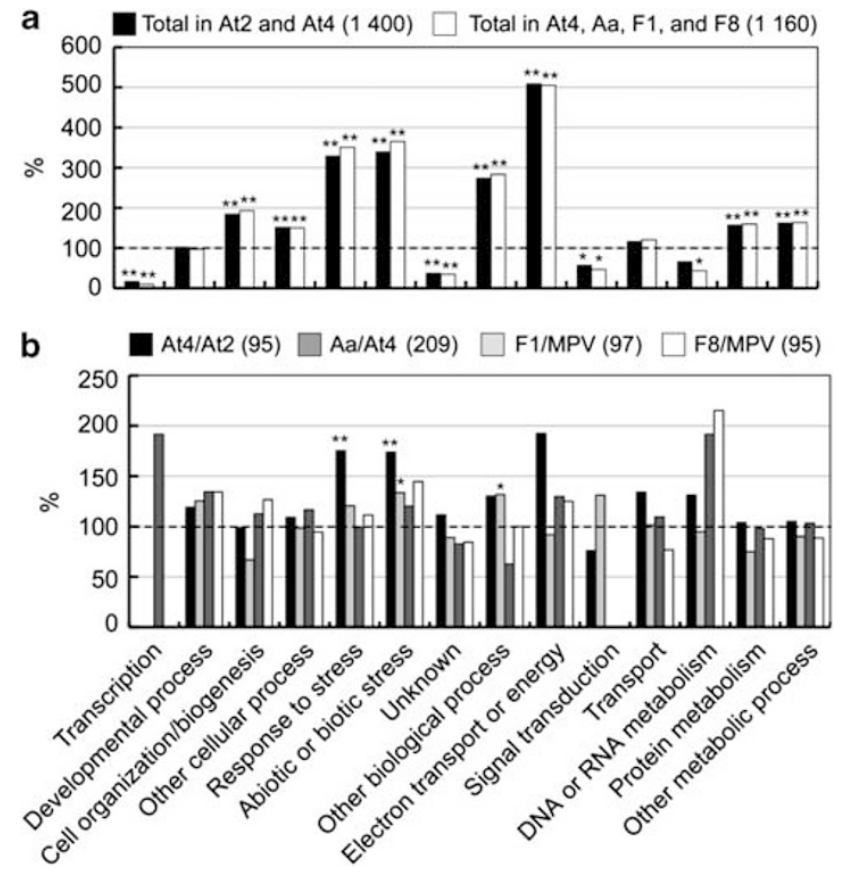

Figure 5 Gene ontology categorization of identified proteins in Arabidopsisrelated species. (a) Total proteins identified by iTRAQ LC-MS/MS analysis were grouped into 14 biological process categories. The percentage of proteins identified in each category was compared with that in the corresponding category of the entire $A$. thaliana genome (dashed line=average or $100 \%$ ). (b) The percentage distribution of differentially or non-additively accumulated proteins in A. thaliana (At2, At4), A. arenosa and resynthesized allotetraploids ( $F_{1}$ and $F_{8}$ generations). The percentage distribution was compared with that of total proteins detected by iTRAQ analysis (dashed line=average or 100\%). MPV: mid-parent value. Significant GO enrichments with $P \leqslant 0.05$ and $P \leqslant 0.01$ were denoted by '*' and '**', respectively.
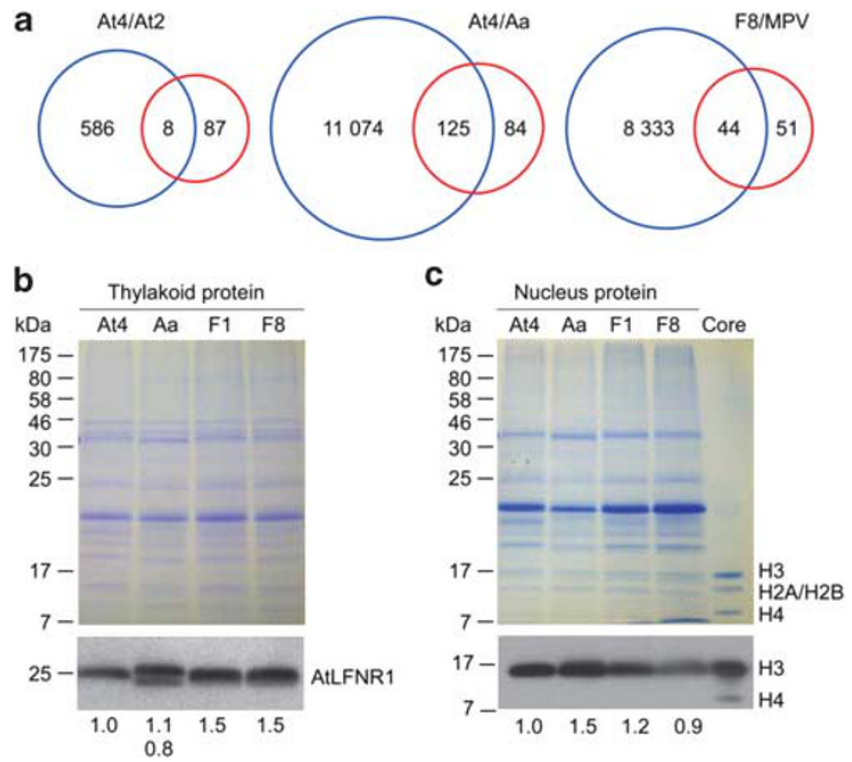

Figure 6 Comparison and validation of proteomic and transcriptomic data. (a) Venn diagrams showing the number of differentially or non-additively expressed proteins in comparison with transcript divergence using per-gene variance in the microarray study (Wang et al., 2006b). (b, c) Validation of protein expression data. (Panel b) Total leaf thylakoid proteins $(20 \mu \mathrm{g})$ extracts from At4, Aa, $F_{1}, F_{8}$ were resolved in a $15 \%$ Urea-SDS-PAGE for subsequent western blot analysis using antibodies against AtLFNR1. (Panel c) The nucleus proteins $(20 \mu \mathrm{g})$ were prepared from the same plant materials as shown in panel $\mathbf{b}$ and used for western blot analysis using the antibodies against histone H3 C terminus. Core histones $(1 \mu \mathrm{g})$ were also included as a positive control. Coomassie blue-stained gels were shown as loading controls. 
( $\sim 40.9 \%$ ) proteins was inversely correlated with the corresponding microarray data in At4/At2, Aa/At4 and $\mathrm{F}_{8} / \mathrm{MPV}$, respectively (Supplementary Tables S2, S3 and S5).

\section{Validation of ITRAQ proteomic data}

In the iTRAQ proteomic analysis, an Arabidopsis leaf-type ferredoxinNADP oxidoreductase (AtLFNR1; At5g66190) was downregulated in $A$. arenosa relative to $A$. thaliana $(\mathrm{Aa} / \mathrm{At} 4=0.82 \pm 0.03$; Supplementary Table S4) and upregulated in $F_{1}(1.55 \pm 0.04)$ and $F_{8}(1.38 \pm 0.04)$ allotetraploids relative to the progenitors (Supplementary Tables S5 and S6). Using the antibodies against AtLFNR1, a specific band was detected in thylakoid protein extracts from At4, AlloF $\mathrm{F}_{1}$ and Allo733F leaves (Figure 6b). Interestingly, a doublet with different sizes was detected in Aa. In allotetraploids, the absence of the corresponding signals similar to that detected in Aa suggested that only the AtLFNR1 homeolog was expressed in allotetraploids. Taking this into consideration, AtLFNR1 intensities in $\mathrm{F}_{1}$ and $\mathrm{F}_{8}$ allotetraploids detected by western blot analysis were consistent with the proteomic data showing higher levels in allotetraploids than the MPV. The different LFNR1 bands and higher signal intensities detected in A. arenosa than in At4 could result from posttranslational modifications and/or presence of a LFNR1 isoform. In addition, sequence divergence between Aa and AtLFNR1 homologs may lead to underestimation of AaLFNR1 peptide levels in the iTRAQ analysis. These factors may explain why the AtLFNR1 ratio between At4 and Aa was lower in iTRAQ assays $(\mathrm{Aa} / \mathrm{At} 4=0.824 \pm 0.029)$ than in the western blot analysis (Figure $6 \mathrm{~b}$ ) or in microarray analysis (Aa/At4 $=1.52$; Supplementary Table S3) (Wang et al., 2006b).

Proteomic data revealed upregulation of histone proteins $(\mathrm{H} 3$ and $\mathrm{H} 4)$ in Aa relative to At4 (Aa/At4 $=2.83 \pm 0.13$ for $\mathrm{H} 3$ and $1.84 \pm 0.18$ for H4) (Supplementary Table S3) and downregulation of histones in allotetraploids $\left(\mathrm{AlloF}_{1}\right.$ or Allo733F $\mathrm{F}_{8}$; Supplementary Tables S4 and S5) relative to the progenitors. Western blot analysis of nucleus extracts using antibodies against histone $\mathrm{H} 3$ indicated the same trend of histone $\mathrm{H} 3$ accumulation levels as that in the proteomic data (Figure 6c).

\section{DISCUSSION}

Proteomic changes between A. thaliana diploid and isogenic autotetraploid are relatively small and enriched in response to stresses

A low level $(\sim 6.8 \%)$ of iTRAQ proteomic difference between Arabidopsis diploids and tetraploids is consistent with the transcriptome data previously reported for Arabidopsis (Wang et al., 2006b), potato (Stupar et al., 2007) and maize (Riddle et al., 2010). The leaf or stem proteomes detected by protein 2-DE were also insignificant between Brassica oleracea diploids and tetraploids (Albertin et al., 2005). The data collectively suggest relatively small changes in gene and protein expression in response to genome doubling (autopolyploidy). Flowcytometry analysis revealed a proportionally higher level of endoreduplication in At4 than that in At2. Thus, proteomic changes observed between A. thaliana diploid and autotetraploid At4 are not likely caused by somatic endoreduplication.

Differentially expressed proteins related to stimuli or stress responses were more enriched in A. thaliana autotetraploids than in diploids. Expression of defense responsive genes often results in a fitness cost to the plants (Zangerl et al., 1997; Heil and Baldwin, 2002; Tian et al., 2003). Constitutive expression of pathogenesis-related genes in Arabidopsis mutants has a negative impact on plant size (Bowling et al., 1997; Clarke et al., 1998). Upregulation of stress and defense responsive genes in $A$. thaliana autotetraploids may thus slow growth. As a result, diploid and autotetraploid plants have a similar size during vegetative growth, despite autotetraploids having generally larger flowers and seeds than diploids (Chen, 2010).

\section{Genome divergence and hybridization have large effects on} proteomic changes

Compared with the relatively small proteomic changes between A. thaliana diploids and autopolyploids, proteomic divergence was relatively high between the two related species, $A$. thaliana and A. arenosa, and their allotetraploids (in $\mathrm{F}_{1}$ and $\mathrm{F}_{8}$ generations). The high level of proteome divergence between $A$. thaliana and $A$. arenosa is consistent with the high level of gene expression divergence detected by microarrays (Wang et al., 2006b) between the two species, which diverged $\sim 6 \mathrm{Mya}$ (Koch et al., 2000). A. thaliana and A. arenosa have five and eight chromosomes in their haploid genomes, respectively. Chromosomal rearrangements such as inversions, translocations and fusions (Kuittinen et al., 2004; Yogeeswaran et al., 2005; Lysak et al., 2006b) may lead to some differences in gene expression and proteomes between the two species. Moreover, cis- and trans-regulatory differences between species (Wang et al., 2006a; Chen, 2007; Wittkopp et al., 2008; $\mathrm{Ng}$ et al., 2011) may also lead to proteomic changes.

In resynthesized allotetraploids, a higher percentage of non-additively accumulated proteins showed downregulation in both $\mathrm{F}_{1}$ and $\mathrm{F}_{8}$ generations, consistent with the observed repression of non-additively expressed genes (approximately 65-76\%) in previous microarray analyses (Wang et al., 2006b). In addition to epigenetic regulation at the transcriptional level, homeologous protein interactions and divergence may also contribute to a high level of divergence in proteomes and regulatory networks between species and in allopolyploids relatively to the parents (Chen, 2007; Doyle et al., 2008; Jackson and Chen, 2010). Moreover, the EF was significantly higher in At4 or Allo $733 \mathrm{~F}_{8}$ than in $\mathrm{Aa}$, suggesting that somatic endopolyploidy may contribute to the high proteome divergence between $A$. arenosa tetraploid and $A$. thaliana autotetraploid lines or resynthesized allotetraploids. On the other hand, proteins that are expressed differently between species may be involved in somatic endopolyploidy levels. Therefore, the effect of somatic endopolyploidy on proteome changes may not be easily separated from that of genetic polyploidy. Interestingly, similar somatic endopolyploidy levels between $A$. thaliana autotetraploid and A. suecica-like allotetraploid suggests that somatic endopolyploidy levels are related to the maternal progenitor A. thaliana. Whether the parent of origin affects somatic endopolyploidy remains to be investigated. In general, the proteomic data suggest larger effects of genome divergence and hybridization on proteomic changes than those of genome doubling (diploid vs tetraploid), consistent with gene expression data (Wang et al., 2006b; Pignatta et al., 2010).

\section{No functional enrichment of non-additively expressed proteins in allotetraploids}

In contrast to autopolyploidization, non-additively expressed proteins in resynthesized allotetraploids $\left(\mathrm{F}_{1}\right.$ or $\mathrm{F}_{8}$ generation) displayed no significant enrichment of particular GO functions. This is similar to the results from synthetic $B$. napus; non-additively expressed proteins were not found to be related to any functional categories or metabolic pathways (Albertin et al., 2007). However, in the transcriptome study, non-additively expressed genes involved in defense and plant hormonal regulation were enriched in Arabidopsis allotetraploids (Wang et al., 2006b). This suggests that interactions between trans-acting homeologous proteins in the same nuclei of allopolyploids may 
provide a mechanism for maintaining the proteomic balance between A. thaliana and A. arenosa genomes. Such regulation at the protein level may compensate changes in transcript levels that are likely affected by cis-regulatory modulation, leading to balanced changes in all categories of proteins. Alternatively, interactions between homeologous proteins could also create new regulatory networks such that functional enrichment of non-additively expressed proteins could only be found under certain growth and environmental conditions. This hypothesis is testable in resynthesized allotetraploids and their progenitors in response to external stimuli.

\section{The degree of changes between transcriptomes and proteomes is similar, but the content of shared changes is different}

Although the levels of transcriptome and proteome divergence were similar in response to genome doubling, divergence or hybridization, the majority of differentially or non-additively expressed proteins were not significantly expressed in microarray analyses (Wang et al., 2006b). A limited correlation between mRNA and protein levels has been reported in yeast (Gygi et al., 1999). In yeast, the aneuploidy effects on transcript and protein levels have also been inconsistent (Torres et al., 2007). The lack of compensation for gene dosage and aneuploidy in yeast may suggest larger effects of aneuploidy than euploidy on transcriptome and proteome, as well as phenotypic variation in response to growth conditions and fitness tests (Pavelka et al., 2010; Springer et al., 2010). A recent study using proteome and transcriptome analysis in single Escherichia coli cells suggested that protein and mRNA copy numbers in single cells for any given gene are uncorrelated, displaying a substantial amount of intrinsic and extrinsic noises (Taniguchi et al., 2010). Comparison of transcriptomes using semiquantitative and quantitative PCR assays and two-dimensional protein gel electrophoresis in $B$. napus also revealed that the majority of transcript changes $(\sim 62 \%)$ did not reflect the differential protein accumulation in the resynthesized allotetraploids compared with the diploid progenitors, B. oleracea and B. rapa (Marmagne et al., 2010). Changes in genome structure, gene expression and interphase organization have also been documented in A. thaliana trisomics (Huettel et al., 2008).

The discordance between transcriptomic and proteomic data is probably related to several factors. First, some gene expression changes are associated with small RNAs, including microRNAs and smallinterfering RNAs (Ha et al., 2009). Moreover, reactivation of retrotransposons in newly synthesized wheat allopolyploids was found to be responsible for sense and antisense transcript production from adjacent genes, leading to subsequent alteration of gene expression in amphiploids (Kashkush et al., 2003). Second, in addition to transcriptional regulation, discordance in gene expression and proteomic changes may reflect transcript stability, post-translational modifications and/or protein stabilities. Third, several experimental factors could contribute to transcriptome and proteome discordance. As examples, the divergence between $A$. arenosa and A. thaliana homologous protein sequences could lead to addition/deletion of either lysine or arginine residues. Therefore, homologous proteins in the $A$. arenosa genome may yield a subset of tryptic-digested peptides that are different from those in the A. thaliana genome. As the International Protein Index Arabidopsis database is built on protein sequences from $A$. thaliana, MS/MS spectra of tryptic-digested peptides originating in the $A$. arenosa genome may be underrepresented in the database. In addition, we cannot exclude a possibility that the discordance is caused by different sets of plant materials used in these comparative studies, as well as the limitation of specificity and sensitivity associated with the microarray technology. Next-generation
mRNA sequencing technology is expected to provide a more accurate and sensitive evaluation of transcriptome changes in A. thalianarelated species and allopolyploids. A more detailed comparative analysis of proteomic and transcriptomic data may help to evaluate the contributions of transcriptional, translational and post-translational regulation of gene expression in Arabidopsis-related species and polyploids. Finally, as mentioned above, expression and proteomic changes in allopolyploids and between species may be confounded by different levels of endoreduplication between A. thaliana and $A$. arenosa and in allotetraploids. Although EFs are proportional to ploidy levels (for example, diploid vs tetraploid) within the same species (A. thaliana), EF factors are different between A. arenosa and other ploidy lines, including allotetraploids. The relative effects of allopolyploidy and endopolyploidy on gene expression and proteomes should be revisited in the future using flow sorting of specific cell types for analyses (Zhang et al., 2005).

\section{Western blot validated proteome data}

Differential accumulation of AtLFNR1 and histone H3 among the Arabidopsis-related species and polyploids detected by the iTRAQ approach were validated by western blot analysis. The same trend of histone $\mathrm{H} 3$ accumulation was detected in At4, Aa, AlloF $F_{1}$ and Allo733F 8 using these two approaches. AtLFNR1 migrated into two distinct spots in two-dimensional protein gel electrophoresis, suggesting possible post-translational modifications of this protein (Lintala et al., 2007), which cannot be detected by microarray analysis in allotetraploids (Wang et al., 2006b). iTRAQ quantitation detected a higher LFNR1 accumulation in A. thaliana than that in A. arenosa because it could not account for the different-size doublet of LFNR1 found in Aa by western blot analysis. In allotetraploids, only the AtLFNR1 homeolog was detected by western blot analysis, and its accumulation level was higher in Aa than in At4. Interestingly, mutation of AtLFNR1 led to a reduced rosette size, chlorophyll content and overall biomass in A. thaliana (Lintala et al., 2007). This may provide a link between AtLFNR1 accumulation and overall increased growth vigor in allotetraploids, demonstrating the importance of comparing transcriptome with proteomic data in studying the consequences of speciation and ploidy effects. Although data validation was very limited at this stage, our results demonstrate that iTRAQ is a valuable approach to the study of proteomic divergence in Arabidopsis-related species and allopolyploids.

\section{CONCLUSIONS}

Complementary to previous transcriptome (Wang et al., 2006b) and small RNA data (Ha et al., 2009), our current proteomic analysis provided a broad view of the rapid and dynamic changes in proteomes in closely related Arabidopsis species and allopolyploids. Genome duplication within A. thaliana resulted in limited changes in proteomes. In contrast but consistent with their evolutionary divergence, autotetraploid $A$. thaliana and outcrossing $A$. arenosa had a relatively high level of proteomic divergence. Differentially expressed proteins between $A$. thaliana diploid and autotetraploid or between $A$. thaliana and $A$. arenosa tetraploids were enriched in functional groups in response to abiotic or biotic stimuli. In contrast, proteomic divergence in allotetraploids relative to the parents was intermediate and occurred over all biological processes. In the future, comparative analyses of these data sets and specific changes in some genes and proteins, as well as genome-wide chromatin modifications (Ha et al., 2011), will allow us to identify additional protein factors and genes that are potentially responsible for altered growth vigor, morphology and adaptation in Arabidopsis species and resynthesized allopolyploids. 


\section{DATA ARCHIVING}

All mass spectra used in this study are publicly available for download at the Proteome Commons Tranche network (https://proteomecom mons.org/tranche/) using the following hash: FZ0WuK7vWJYYFb2qLL7VcZGGV51/YesB2So0SRxp16o34iXM29uQRhAnXu28Ha X9MWEOb9ZSZf5heeYBQ3UIUwL7zjkAAAAAAABZSw==.

A complete listing of protein quantification data is provided as Supplementary data set 1 and it is available for download at the Dryad repository (http://datadryad.org): doi:10.5061/dryad.sk318rd3.

\section{CONFLICT OF INTEREST}

The authors declare no conflict of interest.

\section{ACKNOWLEDGEMENTS}

We thank James A Birchler for the fluorescence in situ hybridization (FISH) cytogenetics workshop where CZ adapted the FISH methodology for this study and Angela Bardo in the Core Facility of Institute for Cellular and Molecular Biology at The University of Texas at Austin for assistance with fluorescence image analysis. We also thank Xiaoli Shi for helpful discussions to improve statistical analysis of the proteomic data. This study was supported by grants from the Plant Genome Research Program of the National Science Foundation (IOS0733857 to ZJC and ISO0924023 to SPB).

Author Contributions: ZJC, DW-KN and CZ designed experiments. DW-KN and $\mathrm{CZ}$ prepared the biological materials and validated the data. $\mathrm{ZS}$ performed iTRAQ proteomic experiments. DW-KN, C Z, ZS, SPB and ZJC analyzed the data. DW-KN and ZJC wrote the paper, and ZS, SPB, DW-KN and ZJC edited the paper.

Albertin W, Alix K, Balliau T, Brabant P, Davanture M, Malosse C et al. (2007). Differential regulation of gene products in newly synthesized Brassica napus allotetraploids is not related to protein function nor subcellular localization. BMC Genomics 8: 56.

Albertin W, Balliau T, Brabant P, Chevre AM, Eber F, Malosse C et al. (2006). Numerous and rapid nonstochastic modifications of gene products in newly synthesized Brassica napus allotetraploids. Genetics 173: 1101-1113.

Albertin W, Brabant P, Catrice O, Eber F, Jenczewski E, Chevre AM et al. (2005). Autopolyploidy in cabbage (Brassica oleracea L.) does not alter significantly the proteomes of green tissues. Proteomics 5: 2131-2139.

Albertin W, Langella O, Joets J, Negroni L, Zivy M, Damerval C et al. (2009). Comparative proteomics of leaf, stem, and root tissues of synthetic Brassica napus. Proteomics 9 . 793-799.

Andreyev AY, Shen Z, Guan Z, Ryan A, Fahy E, Subramaniam S et al. (2010). Application of proteomic marker ensembles to subcellular organelle identification. Mol Cell Proteomics 9: 388-402.

Benjamini Y, Hochberg Y (1995). Controlling the false discovery rate: a practical and powerful approach to multiple testing. J Royal Stat Soci (Series B) 57: 289-300.

Berardini TZ, Mundodi S, Reiser L, Huala E, Garcia-Hernandez M, Zhang P et al. (2004). Functional annotation of the Arabidopsis genome using controlled vocabularies. Plant Physiol 135: 745-755.

Birchler Ja, Newton KJ (1981). Modulation of protein-levels in chromosomal dosage series of maize - the biochemical basis of aneuploid syndromes. Genetics 99: 247-266.

Bowling SA, Clarke JD, Liu Y, Klessig DF, Dong X (1997). The cpr5 mutant of Arabidopsis expresses both NPR1-dependent and NPR1-independent resistance. Plant Cell 9: 1573-1584.

Boyes DC, Zayed AM, Ascenzi R, McCaskill AJ, Hoffman NE, Davis KR et al. (2001). Growth stage-based phenotypic analysis of Arabidopsis: a model for high throughput functional genomics in plants. Plant Cell 13: 1499-1510.

Buggs RJ, Doust AN, Tate JA, Koh J, Soltis K, Feltus FA et al. (2009). Gene loss and silencing in Tragopogon miscellus (Asteraceae): comparison of natural and synthetic allotetraploids. Heredity 103: 73-81.

Carvajal-Rodriguez A, de Una-Alvarez J, Rolan-Alvarez E (2009). A new multitest correction (SGoF) that increases its statistical power when increasing the number of tests. $B M C$ Bioinformatics 10: 209.

Chen ZJ (2007). Genetic and epigenetic mechanisms for gene expression and phenotypic variation in plant polyploids. Annu Rev Plant Biol 58: 377-406.

Chen ZJ (2010). Molecular mechanisms of polyploidy and hybrid vigor. Trends Plant Sci 15: 57-71

Clarke JD, Liu Y, Klessig DF, Dong X (1998). Uncoupling PR gene expression from NPR1 and bacterial resistance: characterization of the dominant Arabidopsis cpr6-1 mutant. Plant Cell 10: 557-569.

Comai L, Tyagi AP, Lysak MA (2003). FISH analysis of meiosis in Arabidopsis allopolyploids. Chromosome Res 11: 217-226.
Comai L, Tyagi AP, Winter K, Holmes-Davis R, Reynolds SH, Stevens Y et al. (2000). Phenotypic instability and rapid gene silencing in newly formed Arabidopsis allotetraploids. Plant Cell 12: 1551-1568.

Cookson SJ, Radziejwoski A, Granier C (2006). Cell and leaf size plasticity in Arabidopsis: what is the role of endoreduplication? Plant Cell Environ 29: 1273-1283.

de Una-Alvarez J, Carvajal-Rodriguez A (2010). 'SGoFicance Trace': assessing significance in high dimensional testing problems. PLoS One 5: e15930.

Diz AP, Carvajal-Rodriguez A, Skibinski DO (2011). Multiple hypothesis testing in proteomics: a strategy for experimental work. Mol Cell Proteomics 10: M110.004374.

Doyle JJ, Flagel LE, Paterson AH, Rapp RA, Soltis DE, Soltis PS et al. (2008). Evolutionary genetics of genome merger and doubling in plants. Annu Rev Genet 42: 443-461.

Flagel L, Udall J, Nettleton D, Wendel J (2008). Duplicate gene expression in allopolyploid Gossypium reveals two temporally distinct phases of expression evolution. BMC Biol 6: 16.

Gaeta RT, Yoo SY, Pires JC, Doerge RW, Chen ZJ, Osborn TC (2009). Analysis of gene expression in resynthesized Brassica napus Allopolyploids using arabidopsis 70mer oligo microarrays. PLoS One 4: e4760.

Galbraith DW, Harkins KR, Maddox JM, Ayres NM, Sharma DP, Firoozabady E (1983). Rapid flow cytometric analysis of the cell cycle in intact plant tissues. Science 220: 1049-1051.

Griffin TJ, Xie H, Bandhakavi S, Popko J, Mohan A, Carlis JV et al. (2007). iTRAQ reagentbased quantitative proteomic analysis on a linear ion trap mass spectrometer. $J$ Proteome Res 6: 4200-4209.

Gygi SP, Rochon Y, Franza BR, Aebersold R (1999). Correlation between protein and mRNA abundance in yeast. Mol Cell Biol 19: 1720-1730.

Ha M, Lu J, Tian L, Ramachandran V, Kasschau KD, Chapman EJ et al. (2009). Small RNAs serve as a genetic buffer against genomic shock in Arabidopsis interspecific hybrids and allopolyploids. Proc Natl Acad Sci USA 106: 17835-17840.

Ha M, Ng DW, Li WH, Chen ZJ (2011). Coordinated histone modifications are associated with gene expression variation within and between species. Genome Res 21: 590-598.

Hegarty MJ, Barker GL, Wilson ID, Abbott RJ, Edwards KJ, Hiscock SJ (2006). Transcriptome shock after interspecific hybridization in senecio is ameliorated by genome duplication. Curr Biol 16: 1652-1659.

Heil M, Baldwin IT (2002). Fitness costs of induced resistance: emerging experimental support for a slippery concept. Trends Plant Sci 7: 61-67.

Holm S (1979). A simple sequentially rejective multiple test procedure. Scand J Stat 6: $65-70$

Hovav R, Udall JA, Chaudhary B, Hovav E, Flagel L, Hu G et al. (2008). The evolution of spinnable cotton fiber entailed prolonged development and a novel metabolism. PLoS Genet 4: e25.

Huettel B, Kreil DP, Matzke M, Matzke AJ (2008). Effects of aneuploidy on genome structure, expression, and interphase organization in Arabidopsis thaliana. PLoS Genet 4: e1000226.

Islam N, Tsujimoto H, Hirano H (2003). Proteome analysis of diploid, tetraploid and hexaploid wheat: towards understanding genome interaction in protein expression. Proteomics 3: 549-557.

Jackson S, Chen ZJ (2010). Genomic and expression plasticity of polyploidy. Curr Opin Plant Biol 13: 153-159.

Kashkush K, Feldman M, Levy AA (2003). Transcriptional activation of retrotransposons alters the expression of adjacent genes in wheat. Nat Genet 33: 102-106.

Kato A, Lamb JC, Birchler JA (2004). Chromosome painting using repetitive DNA sequences as probes for somatic chromosome identification in maize. Proc Natl Acad Sci USA 101: 13554-13559.

Koch MA, Haubold B, Mitchell-Olds T (2000). Comparative evolutionary analysis of chalcone synthase and alcohol dehydrogenase loci in Arabidopsis, Arabis, and related genera (Brassicaceae). Mol Biol Evol 17: 1483-1498.

Koch MA, Wernisch M, Schmickl R (2008). Arabidopsis thaliana's wild relatives: an updated overview on systematics, taxonomy and evolution. Taxon 57: 933-943.

Kuittinen H, de Haan AA, Vogl C, Oikarinen S, Leppala J, Koch M et al. (2004). Comparing the linkage maps of the close relatives Arabidopsis lyrata and $A$. thaliana. Genetics 168: $1575-1584$.

Leitch AR, Leitch IJ (2008). Genomic plasticity and the diversity of polyploid plants. Science 320: 481-483.

Lintala M, Allahverdiyeva Y, Kidron H, Piippo M, Battchikova N, Suorsa M et al. (2007). Structural and functional characterization of ferredoxin-NADP+-oxidoreductase using knock-out mutants of Arabidopsis. Plant J 49: 1041-1052.

Lysak M, Fransz P, Schubert I (2006a). Cytogenetic analyses of Arabidopsis. Methods Mol Biol 323: 173-186

Lysak MA, Berr A, Pecinka A, Schmidt R, McBreen K, Schubert I (2006b). Mechanisms of chromosome number reduction in Arabidopsis thaliana and related Brassicaceae species. Proc Natl Acad Sci USA 103: 5224-5229.

Marmagne A, Brabant P, Thiellement H, Alix K (2010). Analysis of gene expression in resynthesized Brassica napus allotetraploids: transcriptional changes do not explain differential protein regulation. New Phytol 186: 216-227.

Ng DW, Zhang Z, Miller M, Palmer G, Whiteley M, Tholl D et al. (2011). Cis- and transregulation of miR 163 and target genes confers natural variation of secondary metabolites in Arabidopsis related species and allopolyploids. Plant Cell 23: 1729-1740.

O'Brien RN, Shen Z, Tachikawa K, Lee PA, Briggs SP (2010). Quantitative proteome analysis of pluripotent cells by iTRAQ mass tagging reveals post-transcriptional regulation of proteins required for ES cell self-renewal. Mol Cell Proteomics 9: 2238-2251.

O'Kane S, Schaal B, Al-Shehbaz I (1995). The origins of Arabidopsis suecica (Brassicaceae), as indicated by nuclear rDNA sequences, and implications for rDNA evolution. System Bot 21: 559-566. 
Pavelka N, Rancati G, Zhu J, Bradford WD, Saraf A, Florens L et al. (2010). Aneuploidy confers quantitative proteome changes and phenotypic variation in budding yeast. Nature 468: 321-325.

Pignatta D, Dilkes BP, Yoo SY, Henry IM, Madlung A, Doerge RW et al. (2010). Differential sensitivity of the Arabidopsis thaliana transcriptome and enhancers to the effects of genome doubling. New Phytologist 186: 194-206.

Riddle NC, Jiang H, An L, Doerge RW, Birchler JA (2010). Gene expression analysis at the intersection of ploidy and hybridity in maize. Theor App/ Genet 120: 341-353.

Ross PL, Huang YN, Marchese JN, Williamson B, Parker K, Hattan S et al. (2004). Multiplexed protein quantitation in Saccharomyces cerevisiae using amine-reactive isobaric tagging reagents. Mol Cell Proteomics 3: 1154-1169.

Saleh A, Alvarez-Venegas R, Avramova Z (2008). An efficient chromatin immunoprecipitation (ChIP) protocol for studying histone modifications in Arabidopsis plants. Nat Protoc 3: $1018-1025$

Salmon A, Ainouche ML, Wendel JF (2005). Genetic and epigenetic consequences of recent hybridization and polyploidy in Spartina (Poaceae). Mol Ecol 14: 1163-1175.

SchmickI R, Koch MA (2011). Arabidopsis hybrid speciation processes. Proc Natl Acad Sci USA 108: 14192-14197.

Schwartz JC, Syka JEP, Quarmby ST (2005). The 53rd ASMS Conference on Mass Spectrometry and Allied Topics. San Anotonio, Texas.

Soltis PS, Soltis DE (2009). The role of hybridization in plant speciation. Annu Rev Plant Biol 60: 561-588.

Song X, Ni Z, Yao Y, Xie C, Li Z, Wu H et al. (2007). Wheat (Triticum aestivum L.) root proteome and differentially expressed root proteins between hybrid and parents. Proteomics 7: 3538-3557.

Springer M, Weissman JS, Kirschner MW (2010). A general lack of compensation for gene dosage in yeast. Mol Syst Biol 6: 368.

Stupar RM, Bhaskar PB, Yandell BS, Rensink WA, Hart AL, Ouyang S et al. (2007). Phenotypic and transcriptomic changes associated with potato autopolyploidization. Genetics 176: 2055-2067.

Taniguchi Y, Choi PJ, Li GW, Chen H, Babu M, Hearn J et al. (2010). Quantifying E. coli proteome and transcriptome with single-molecule sensitivity in single cells. Science 329: 533-538.
Tate JA, Ni Z, Scheen AC, Koh J, Gilbert CA, Lefkowitz D et al. (2006). Evolution and expression of homeologous Loci in Tragopogon miscellus (Asteraceae), a recent and reciprocally formed allopolyploid. Genetics 173: 1599-1611.

Tian D, Traw MB, Chen JQ, Kreitman M, Bergelson J (2003). Fitness costs of R-genemediated resistance in Arabidopsis thaliana. Nature 423: 74-77.

Torres EM, Sokolsky T, Tucker CM, Chan LY, Boselli M, Dunham MJ et al. (2007). Effects of aneuploidy on cellular physiology and cell division in haploid yeast. Science $\mathbf{3 1 7}$ : 916-924.

Wang J, Tian L, Lee HS, Chen ZJ (2006a). Nonadditive regulation of FRI and FLC Loci Mediates flowering-time variation in Arabidopsis allopolyploids. Genetics 173: 965-974.

Wang J, Tian L, Lee HS, Wei NE, Jiang H, Watson B et al. (2006b). Genome-wide nonadditive gene regulation in Arabidopsis allotetraploids. Genetics 172: 507-517.

Wang J, Tian L, Madlung A, Lee HS, Chen M, Lee JJ et al. (2004). Stochastic and epigenetic changes of gene expression in Arabidopsis polyploids. Genetics 167: 1961-1973.

Wendel JF (2000). Genome evolution in polyploids. Plant Mol Biol 42: 225-249.

Wittkopp PJ, Haerum BK, Clark AG (2008). Regulatory changes underlying expression differences within and between Drosophila species. Nat Genet 40: 346-350.

Wood TE, Takebayashi N, Barker MS, Mayrose I, Greenspoon PB, Rieseberg LH (2009). The frequency of polyploid speciation in vascular plants. Proc Natl Acad Sci USA 106: $13875-13879$

Yao H, Kato A, Mooney B, Birchler JA (2011). Phenotypic and gene expression analyses of a ploidy series of maize inbred Oh43. Plant Mol Biol 75: 237-251.

Yogeeswaran K, Frary A, York TL, Amenta A, Lesser AH, Nasrallah JB et al. (2005). Comparative genome analyses of Arabidopsis spp.: inferring chromosomal rearrangement events in the evolutionary history of $A$. thaliana. Genome Res 15: 505-515.

Zangerl AR, Arntz AM, Berenbaum MR (1997). Physiological price of an induced chemical defense: photosynthesis, respiration, biosynthesis, and growth. Oecologia 109: 433-441.

Zhang C, Gong FC, Lambert GM, Galbraith DW (2005). Cell type-specific characterization of nuclear DNA contents within complex tissues and organs. Plant Methods 1: 7.

Supplementary Information accompanies the paper on Heredity website (http://www.nature.com/hdy) 